\title{
MICROCOMPUTER CHESS: HAS IT REACHED A NEW MILESTONE?
}

\author{
ICCA Communication \\ by Stuart Cracraft \\ UCLA, Los Angeles, CA
}

Recent advances in hardware architecture have resulted in yet another leap for microcomputer chess. Formerly stymied at approximately the class A level (USCF rating 1800 to 2000), there is strong evidence to support the contention that there now exist commercially available microcomputer-chess machines that break into the candidate-master category (USCF rating 2000 to 2200), and perhaps even low master category (USCF 2200). This article supplies data to support the above assertion.

On the weekend of February 14th and 15th, 1987, the U.S. Amateur Team Tournament was held in Pasadena, California. This particular event allows teams of four players to play a six-round Swiss-style tournament. The computer manufacturer Novag entered four of its commercially available machines. The Novag machines were Super Constellation (at $6.0 \mathrm{Mhz}$ ), Forte (at $5.5 \mathrm{Mhz}$ ), Expert (at $6.0 \mathrm{Mhz}$ ), and Turbo (at 16.0 Mhz). Two of these machines, Super Constellation and Expert, are well-known and have been available to the public for quite some time. The other two entires, Forte and Turbo, represent fairly recent additions to Novag's product line.

The result of the tournament was interesting. In the table reproduced below, the data represent the result of the outcome from the machine's point of view and the rating of the opponent.

\begin{tabular}{|c|rr|rr|rr|rr|}
\hline Board Number & & $(1)$ & & $(2)$ & \multicolumn{1}{c|}{$(3)$} & $(4)$ \\
\hline Round Numbers & & Expert & & Turbo & & Super-C & Forte \\
\hline 1 & 1 & 1615 & 1 & 1820 & 1 & 1850 & $1 / 2$ & 1900 \\
2 & 0 & 2396 & 1 & 2117 & 0 & 2089 & 1 & 2026 \\
3 & 0 & 2369 & 1 & 2108 & $1 / 2$ & 2103 & $1 / 2$ & 2075 \\
4 & 0 & 2217 & 1 & 2126 & 0 & 2105 & $1 / 2$ & 2067 \\
5 & 1 & 2115 & 1 & 1908 & $1 / 2$ & 1789 & 0 & 1563 \\
6 & $1 / 2$ & 2071 & 1 & 2057 & 0 & 1996 & 0 & 1897 \\
\hline TOTAL SCORE & $21 / 2$ & & 6 & & 2 & & 3 & \\
\hline Average Rating of Opponents & \multicolumn{3}{|c|}{2130} & & 2023 & & 1989 & 1927 \\
\hline Performance Rating of Machine & \multicolumn{3}{|c|}{2072} & 2423 & & 1872 & 1927 \\
\hline
\end{tabular}

Some additional information is useful in evaluating this table. (1) Turbo was placed on board 2 rather than board 1 due to its being an experimental machine with relatively little previous practical experience on which to judge its strength. (2) Against master opponents, no points or half-points were won. But only three such games were played, all by the Expert. (3) Against expert opponents, in twelve games, a total of eight points were won for a winning percentage of $67 \%$. (4) Against lower-rated opponents, a total of ten games were played and six points were won, for a total winning percentage of $60 \%$. (5) Forte's result may be understated because of time-control problems resulting from one person operating all four machines at once.

The most remarkable result of this tournament, obviously, belongs to the Turbo, an advanced, bitsliced, 6502-based processor-design that is now commercially available. Bit-slicing involves the redesign of single processors using multiple, faster chips. Turbo's six-win clean sweep against strong opposition is remarkable. This machine has also played a 10-game speed match against USCF Senior Master Jeremy Silman and won 7.5 to 2.5 against him. 
In evaluating the Turbo's result and estimating its strength, it is important to realize that it is exactly four times as fast as the original 4 Megahertz version of the Novag Expert. Additionally, it searches approximately 3000-4000 chess positions per second. The author performed a test that showed the Turbo searched the opening position of a chess game to a depth of six ply exactly four times as fast as the Expert which ran at 4 Megahertz, thus confirming the manufacturer's claims.

A rough rule-of-thumb by chess researchers over the years is that if a machine doubles in processor speed, an approximate 100 point rating increase will result. This relationship is true in the range up to 2000 rating points. According to some later results, the increase is not quite 100 points for ranges beyond 2000 rating points. However, this is a point of contention among researchers and there is no general agreement. For our purposes, 100 points per doubling is a good estimate. Since the Turbo is four times as fast as the Expert, one would expect it to be perhaps 200 points stronger, a full class.

The Novag Expert $4 \mathrm{Mhz}$ is rated on the Swedish Rating List (published in the ICCA Journal, Vol. 9, No. 4, December 1986) as having an ELO rating of 1845, based on 371 games played against human competition, with the Novag Expert $4 \mathrm{Mhz}$ holding a 52\% edge against opponents who had an average rating of 1831. This would roughly correspond to a USCF rating of 1945 and corresponds with my own evaluation of the Novag Expert in a twenty-game match between it and myself. The Expert showed an approximate $90 \%$ winning percentage over me, a provisionally-rated player of 1553 . This translates to a rating of 1919 , closely approximating the Swedish rating. Also, the latest USCF rating of the Expert $6 \mathrm{Mhz}$ (50\% faster than the Expert $4 \mathrm{Mhz}$ ) is 2106.

Since the Expert $4 \mathrm{Mhz}$ is rated as a strong A player (USCF 1919-1945), we can now calculate the estimated rating of the Turbo as one full class ahead of the Expert $4 \mathrm{Mhz}$. This would translate to a USCF 2119-2145 rating, strong candidate master, an excellent result for the Turbo. Turbo's programmer, David Kittinger, estimates that Turbo is a "weak master" based on master results of a less-speedy version of the commercially available Turbo. His estimate, while somewhat higher than our own, is reasonable. Additional tournament results for the Turbo will supply a more definite rating. At the very least, Turbo (along with Mephisto's top-of-the-line machine) is the strongest commercially-marketed machine ever.

We now reproduce the six games played by the Turbo at the Pasadena tournament.

Round 1, Hwang (White) vs. Novag Turbo (Black), USCF 1820

1. d4 Nf6 2. e4 Nxe4 3. Qf3 d5 4. Bd3 c5 5. dxc5 Nxc5 6. b3 Nxd3+ 7. Qxd3 e5 8. Bb2 Bb4+ 9. Nd2 Qg5 10. Ngf3 Qxg2 11. Rg1 Bxd2+ 12. Kxd2 Qxf2+ 13. Qe2 Qxe2+ 14. Kxe2 0-0 15. Nxe5 f6 16. Nd3 Nc6 17. Rg3 Bd7 18. Raf1 Rae8+ 19. Kd2 Ne5 20. Nf4 Bc6 21. Nh5 Ng6 22. h4 Re6 23. Nxf6 gxf6 24. h5 Kf7 25. hxg6 hxg6 26. Ba3 Rfe8 27. Rh3 Re2+ 28. Kc1 f5 29. Rh7+ Kg8 30. Rhh1 d4 31. Rhg1 Be4 32. Rxg6+ Kf7 33. Rfg1 Rxc2+ 34. Kd1 Rxa2 35. Rg7+ Ke6 36. R1g6+ Ke5 37. Bd6+ Kd5 38. Bb4 Bc2+ 39. Kc1 Bxb3 40. Rd6+ Kc4 41. Rxb7 Ree2 42. Rc6+ Kd3 0-1

Round 2, Novag Turbo (White) vs. Pell (Black), USCF 2117

1. e4 e5 2. Nf3 Nf6 3. Nxe5 d6 4. Nf3 Nxe4 5. d4 d5 6. Bd3 Be7 7. 0-0 Nc6 8. c4 Nb4 9. cxd5 Nxd3 10. Qxd3 Qxd5 11. Nc3 Nxc3 12. Qxc3 c6 13. Re1 Be6 14. Bf4 0-0 15. Re5 Qd7 16. Re3 Rad8 17. Rae1 Rie8 18. h3 Qd5 19. a3 c5 20. dxc5 Qxc5 21. Ng5 Qxc3 22. Rxc3 Bf6 23. Rc2 Bd5 24. Re3 h6 25. Nf3 a6 26. Be5 Bxe5 27. Nxe5 f6 28. Nc4 Kf7 29. Rxe8 Kxe8 30. Nb6 Bc6 31. f3 Rd6 32. Kf2 Re6 33. Rc3 Kf7 34. Re3 Ke7 35. Rxe6+ Kxe6 36. Ke3 Kd6 37. Kd4 Bb5 38. Nc4 Ke6 39. Kc5 Bxc4 40. Kxc4 b6 41. Kd4 1-0 (time forfeit)

Round 3, Saints (White) vs. Novag Turbo (Black) USCF 2108

1. e4 c5 2. Nc3 Nc6 3. g3 g6 4. Bg2 Bg7 5. f4 Nf6 6. d3 0-0 7. Nf3 d5 8. e5 Ng4 9. 0-0 a6 10. Qe2 Rb8 11. Rb1 b6 12. Ng5 Nd4 13. Qd1 h6 14. Nf3 Nf5 15. Qe2 Be6 16. Bh3 Nxe5 17. Nxe5 Nxg3 18. hxg3 Bxh3 19. Nc6 Bxf1 20. Kxf1 Qd7 21. Nxe7+ Kh7 22. Nexd5 b5 23. Qf3 Rfd8 24. Ne3 Re8 25. Bd2 c4 26. Ncd5 cxd3 27. cxd3 Rbd8 28. Ba5 Rxe3 29. Nxe3 Qxd3+ 30. Kg2 Qxb1 31. Bxd8 Qxa2 32. Qf2 Bd4 33. Kf3 Qe6 34. Ba5 Qc6+ 35. Ke2 b4 36. Kd2 Bxe3 37. Qxe3 Qd5+ 38. Kc2 Qxa5 39. Qa7 Qa4+ 40. b3 Qa2+ 41. Kc1 Qxb3 42. Qxa6 Qa3+ 43. Qxa3 bxa3 44. Kb1 f5 45. Ka2 g5 46. fxg5 hxg5 $0-1$ 
Round 4, Novag Turbo (White) vs. Ludwinski (Black), USCF 2126

1. e4 e5 2. Nf3 Nf6 3. Nxe5 d6 4. Nf3 Nxe4 5. d4 d5 6. Bd3 Bd6 7. c4 Bg4 8. 0-0 f5 9. cxd5 0-0 10. Nc3 Qe7 11. h3 Bh5 12. Nb5 Nd7 13. Re1 Rae8 14. Bg5 Qxg5 15. Nxg5 Bxd1 16. Bxe4 fxe4 17. Raxd1 Nf6 18. Nc3 Bb4 19. Ngxe4 Nxe4 20. Rxe4 Bxc3 21. Rxe8 Rxe8 22. bxc3 Re2 23. Rb1 b6 24. Rb4 Kf7 25. Kf1 Rxe7 26. Rb1 h6 27. c4 Re4 28. Rd1 Rh4 29. f3 Ke7 30. Kf2 Kd6 31. a4 a6 32. Kg3 g5 33. Rd2 b5 34. axb5 axb5 35. cxb5 Kxd5 36. Rc2 Kd6 37. Rc6+ Kd7 38. Rc4 Rf4 39. Kf2 Rf8 40. g4 Ra8 41. Rc6 Rh8 42. Kg3 Rb8 43. Rc5 Kd6 44. f4 gxf4+ 45. Kxf4 Rf8+ 46. Ke4 Rh8 47. Kd3 Kd7 48. Kc4 Ra8 49. Rh5 Rh8 50. g5 Rg8 51. gxh6 1-0

Round 5, Parrott (White) vs. Novag Turbo (Black), USCF 1908

1. e4 c5 2. Nc3 Nc6 3. f4 g6 4. Nf3 Bg7 5. g3 d6 6. Bg2 Nf6 7. d3 0-0 8. 0-0 d5 9. Kh1 d4 10. Ne2 b5 11. c3 Bb7 12. Qc2 Qb6 13. h3 Rad8 14. Bd2 Nd7 15. g4 f5 16. Qb3+ Kh8 17. gxf5 gxf5 18. Ng5 fxe4 19. Bxe4 c4 20. dxc4 h6 21. Qxb5 hxg5 22. Qxg5 Rf6 23. Rg1 Bh6 24. Qh5 Nce5 25. fxe5 Bxe4+ 26. Kh2 dxc3 27. Be1 Rf2+ 29. Kg3 cxb2 29. Bxf2 Rg8+ 0-1

Round 6, Novag Turbo (White) vs Hodges (Black), USCF 2057

1. e4 e6 2. d3 d5 3. Nd2 Nf6 4. g3 b6 5. Bg2 Bb7 6. e5 Nfd7 7. Ngf3 c5 8. 0-0 Nc6 9. Re1 Qc7 10. Qe2 Be7 11. c3 h6 12. Nf1 g5 13. h3 0-0-0 14. b4 cxb4 15. cxb4 Bxb4 16. Bd2 Ba6 17. Bxb4 Nxb4 18. Rec1 Nc5 19. Ne1 Bxd3 20. Qb2 Bxf1 21. Bxf1 Nc6 22. Nd3 Kd7 23. Qa3 Nxd3 24. Bxd3 Qxe5 25. Bb5 Rc8 26. Qxa7+ Rc7 27. Bxc6+ Ke7 28. Qa3+ Kf6 29. Re1 Qd4 30. Rad1 Qc5 31. Qb2+ d4 32. Rxd4 e5 33. Rxe5 Qxc6 34. Rd6+ Qxd6+ 35. Rd5+ Ke7+ 36. Rxd6 Kd6 37. Qxh8 Rc1+ 38. Kg2 Ke6 39. Qxh6 f6 40. h4 Rc5 41. a4 gxh4 42. Qh4 b5 44. Qe4+ Kd6 44. Qd4+ Rd5 45. Qxf6+ Kc5 46. Qe7+ Kb6 47. Qe6+ Kc5 48. axb5 Rd3 49. Qe7+ Kd4 50. Qa7+ Ke5 51. b6 1-0

Thanks are due Rainer Rickford, David Kittinger, and David Hough for their assistance during the preparation of this contribution.



$\mathrm{KBBKN}$ or :

Kasparov Being Baffled - Knowledge Nichevo 